\title{
Herbal Substance, Acteoside, Alleviates Intestinal Mucositis in Mice
}

\author{
Daniel Reinke, ${ }^{1,2}$ Stamatiki Kritas, ${ }^{1}$ Panagiotis Polychronopoulos, ${ }^{3}$ Alexios L. Skaltsounis, ${ }^{3}$ \\ Nektarios Aligiannis, ${ }^{3}$ and Cuong D. Tran ${ }^{1,2}$ \\ ${ }^{1}$ Gastroenterology Unit, Women's \& Children's Health Network, 72 King William Road, North Adelaide, SA 5006, Australia \\ ${ }^{2}$ Discipline of Physiology, School of Medical Sciences, University of Adelaide, Adelaide, SA 5005, Australia \\ ${ }^{3}$ Department of Pharmacognosy \& Natural Products Chemistry, National and Kapodistrian University of Athens, 15771 Ilisia, Greece
}

Correspondence should be addressed to Cuong D. Tran; cuong.tran@adelaide.edu.au

Received 21 October 2014; Revised 4 December 2014; Accepted 4 December 2014

Academic Editor: Gianfranco D. Alpini

Copyright (C) 2015 Daniel Reinke et al. This is an open access article distributed under the Creative Commons Attribution License, which permits unrestricted use, distribution, and reproduction in any medium, provided the original work is properly cited.

This study investigated the role of acteoside in the amelioration of mucositis. C57BL/6 mice were gavaged daily with acteoside $600 \mu \mathrm{g}$ for $5 \mathrm{~d}$ prior to induction of mucositis and throughout the experimental period. Mucositis was induced by methotrexate (MTX; $12.5 \mathrm{mg} / \mathrm{kg}$; s.c.). Mice were culled on d 5 and d 11 after MTX. The duodenum, jejunum, and ileum were collected for myeloperoxidase (MPO) activity, metallothionein (MT) levels, and histology. Acteoside reduced histological severity scores by 75,78 , and $88 \%$ in the duodenum, jejunum, and ileum, respectively, compared to MTX-controls on d 5 . Acteoside reduced crypt depth by 49, 51, and $33 \%$ and increased villus height by 19,38 , and $10 \%$ in the duodenum, jejunum, and ileum, respectively, compared to MTX-controls on d 5 . Acteoside decreased MT by $50 \%$ compared to MTX-control mice on d 5 . Acteoside decreased MPO by $60 \%$ and $30 \%$ in the duodenum and jejunum, respectively, compared to MTX-controls on d 5. Acteoside alleviated MTX-induced small intestinal mucositis possibly by preventing inflammation.

\section{Introduction}

Mucositis is characterised by the degradation and increased ulceration of the protective mucosal layer within the gastrointestinal tract (GIT) [1] and occurs in over $40 \%$ of all cancer patients that undergo chemotherapy or radiotherapy. Chemotherapeutic agents, such as methotrexate (MTX), attack rapidly dividing cancer cells by inhibiting folate production in RNA and DNA synthesis [1-3]. However, these agents are unable to differentiate between all rapidly dividing cells resulting in destruction of the basal cells and damage to connective tissue in the GIT [3].

Damage to the intestinal lining causes debilitating symptoms $[4,5]$. These symptoms are divided into two categories, pain and inflammation. Pain symptoms include loss of eating and eventual anorexia [6]. Inflammation symptoms include diarrhoea, vomiting, and reduced nutrient uptake [7].

The current treatments for mucositis are systemic antibiotics [7-9], anti-inflammatories such as benzydamine and flurbiprofen [7], reducing the immune response and inflammatory markers present in the small intestine $[5,10]$, and nonspecific pain killers [8] which reduce the amount of damage signalling by nociceptors [11]. Antibiotics decrease levels of bacteria in the small intestine reducing immune mediated inflammation responses [7-9]. These treatments only mask the symptoms but do not treat the condition.

While there are currently no effective treatments for mucositis, many are in development. Numerous novel compounds which may provide preventative protection are being examined, for example, growth factors such as palifermin $[7,8]$, free radical scavengers such as amifostine $[7,8]$, or herbal substance such as iberogast [12] and Rhodiola algida [13].

The herbal substance, acteoside, is a phenylpropanoid glycoside that is derived from plant species in the Scrophularia genus $[14,15]$. Plants containing acteoside are often used in traditional medicine to treat a variety of illnesses, such as tumours [16] and inflammation [17]. Hausmann et al. [18] 


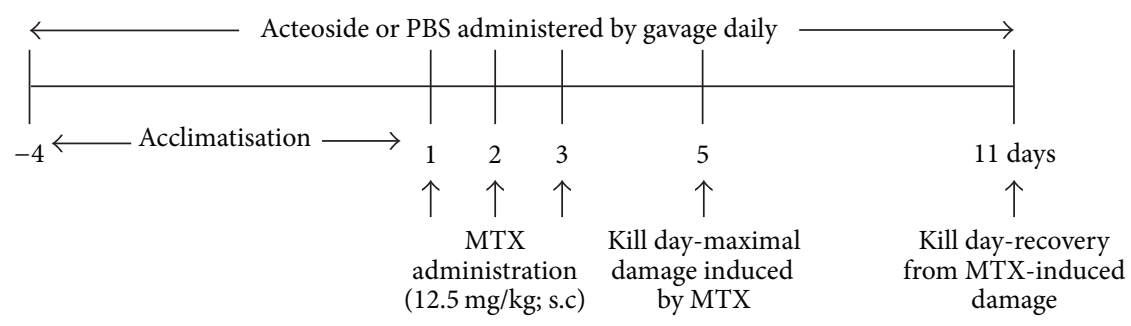

FIGURE 1: Schematic diagram of the experimental design.

demonstrated that acteoside reduced the intestinal damage caused by experimentally induced inflammatory bowel disease. Acteoside may exert its protective effect through a variety of mechanisms. It is known as an antioxidant [17] that prevents cell apoptosis $[19,20]$ and sequesters reactive oxygen species (ROS) [21].

It is hypothesised that prophylactic treatment of mice with acteoside will prevent damage caused by MTX to the intestinal mucosa. In the present study, we will be utilising a C57BL/6-mouse model of MTX-induced mucositis to examine the efficacy of acteoside as a potential treatment of mucositis. The mouse model of MTX-induced intestinal mucositis is a well-established model allowing the formation of well-defined, reproducible, clinical, and histological symptoms of mucositis [22].

\section{Materials and Methods}

2.1. Animals and Diet. All animal studies were performed in compliance with the Australian Code of Practice for the Care and Use of Animals for Scientific Purposes and were approved by the Animal Ethics Committee of the Women's and Children's Health Network (WCHN). Male C57BL/6 mice ( $n=64,7$ weeks of age) were housed at room temperature maintained at 25 degrees Celsius with 12-hour dark and light cycles. Mice were given access to standard chow pellets (Specialty Feeds, WA, Australia) and free access to water.

2.2. Study Design. The experiment was undertaken for $16 \mathrm{~d}$, between $\mathrm{d}-4$ and $\mathrm{d} 11$. The first MTX injection was given on $\mathrm{d} 1$. As maximum damage to the small intestinal lining occurs $5 \mathrm{~d}$ after the first injection of MTX, mice were culled to assess prevention of damage. Recovery from MTX-induced gut damage occurs from $11 \mathrm{~d}$ after the first MTX injection and so this was selected as a cull day to assess the ability of acteoside to enhance intestinal recovery (Figure 1).

2.3. Disease Activity Index. The animal's health was recorded using the standard disease activity index (DAI) scoring system and their body weight was recorded daily. DAI is a representative score given for weight loss, general well-being, stool consistency, and blood content in the stool. Categories are rated 1 to 4 , with $1=$ normal and $4=$ severe. Scores from the overall categories are combined for a daily DAI score.

2.4. Acteoside, MTX Preparation and Administration. Acteoside (provided by Kapodistrian University of Athens, Greece) powder with purity of $>97 \%$ was used to prepare a stock solution by suspending $6000 \mu \mathrm{g}$ dried powder in $1 \mathrm{~mL}$ of phosphate buffered saline (PBS). Acteoside $(600 \mu \mathrm{g})$ was gavage $(0.1 \mathrm{~mL}$ of $6000 \mu \mathrm{g} / \mathrm{mL})$ daily throughout the experimental period (Figure 1).

MTX (12.5 mg/kg) was diluted from $50 \mathrm{mg} / \mathrm{mL}$ solution (Pfizer Australia Pty Ltd, NSW, Australia) and was injected subcutaneously daily for 3 consecutive days into mice to induce mucositis (Figure 1). Treatments were given to the respective groups by oral gavage. Mice were randomly allocated to 4 groups: PBS + Saline (PS; $n=16)$, Acteoside + Saline (AS; $n=16)$, PBS + MTX (PM; $n=16)$, and Acteoside $+\operatorname{MTX}(\mathrm{AM} ; n=16)$.

2.5. Tissue Collection. Mice were culled by asphyxiation with $\mathrm{CO}_{2}$ followed by cervical dislocation on $\mathrm{d} 5$ and $\mathrm{d} 11$, after MTX injections (Figure 1). Tissue samples were collected from the duodenum, jejunum, and ileum for histology and biochemical assays (these samples were snap frozen in liquid nitrogen and then stored at $-80^{\circ} \mathrm{C}$ until analysis).

2.6. Myeloperoxidase (MPO) Activity. MPO activity in intestinal tissues was determined using a modification of the method by Dahlqvist [23]. Briefly, samples were homogenised (Ultra Turrex homogeniser, Janke and Kunkel, Germany) in $2 \mathrm{~mL}$ of saline and centrifuged for $12 \mathrm{~min}$ at $15000 \mathrm{rpm}$ (Mikro Benchtop Centrifuge, Hettich $\mathrm{GmbH}$ and Co., Tuttlingen, Germany). The supernatant was discarded and the pellet was resuspended in hexadecyltrimethylammonium bromide buffer (HTAB), a detergent (Sigma Aldrich, Sydney, Australia). $50 \mu \mathrm{L}$ of negative and positive control samples as well as $50 \mathrm{ul}$ of each test sample was added to triplicate wells in a microtiter plate. $200 \mathrm{uL}$ of reaction solution, consisting of $8.4 \mathrm{mg}$ of O-dianisidine dihydrochloride, $24 \mathrm{uL}$ of $\mathrm{H}_{2} \mathrm{O}_{2}, 5 \mathrm{~mL}$ of potassium phosphate buffer $(\mathrm{pH} 6.0$ ) and $45 \mathrm{~mL}$ of distilled $\mathrm{H}_{2} \mathrm{O}$, was added to all wells in the plate and absorbance was read at $450 \mathrm{~nm}$ at 1 min intervals for $15 \mathrm{~min}$ with a spectrophotometer (Dynatech MR700, Baxter Diagnostics, Adelaide, South Australia). Results were expressed as units/g of tissue.

2.7. Metallothionein (MT) Assay. MT levels in intestinal tissues were determined using a radioactivity ${ }^{109} \mathrm{Cd} / \mathrm{haem}$ affinity assay as described previously [24]. Briefly, intestinal tissues were homogenized in $10 \mathrm{mM}$ Tris- $\mathrm{HCl}(\mathrm{pH} \mathrm{8.2)}$ and heated in a boiling water bath for $2 \mathrm{~min}$ and then spun at $12,000 \mathrm{~g}$ for $2 \mathrm{~min}$. The supernatant was diluted $(2: 1)$ in $10 \mathrm{mM}$ Tris-HCL and ${ }^{109} \mathrm{Cd}$ (Oak Ridge National Laboratory, 
TABLE 1: Disease activity index of C57BL/6 mice ( $n=8$ /group) treated with PS, PM, AS, and MA.

\begin{tabular}{|c|c|c|c|c|c|c|}
\hline Treatment & $\mathrm{d}-4$ & $\mathrm{~d} 1$ & $\mathrm{~d} 2$ & d 3 & d 5 & $\mathrm{~d} 11$ \\
\hline PS & $1(1,1)$ & $2(1,2)$ & $1(1,2)$ & $1(1,2)$ & $1(1,2)$ & $1(1,1)$ \\
\hline AS & $1(1,1)$ & $1(1,2)$ & $2(1,4)$ & $1.5(1,2)$ & $1(1,1)$ & $1(1,1)$ \\
\hline $\mathrm{PM}$ & $1(1,1)$ & $2(1,2)$ & $2(1,4)$ & $3(2,4)^{*}$ & $2(1,3)^{*}$ & $1(1,1)$ \\
\hline MA & $1(1,1)$ & $1(1,2)$ & $2(1,4)$ & $2(1,3)$ & $1.5(1,2)$ & $1(1,1)$ \\
\hline
\end{tabular}

Data was expressed as median and range in parenthesis. ${ }^{*}$ Statistical significance $P=0.01$ compared to PS within group. PS $=$ PBS + saline, AS $=$ acteoside + saline, PM = PBS + MTX, and MA = MTX + acteoside.

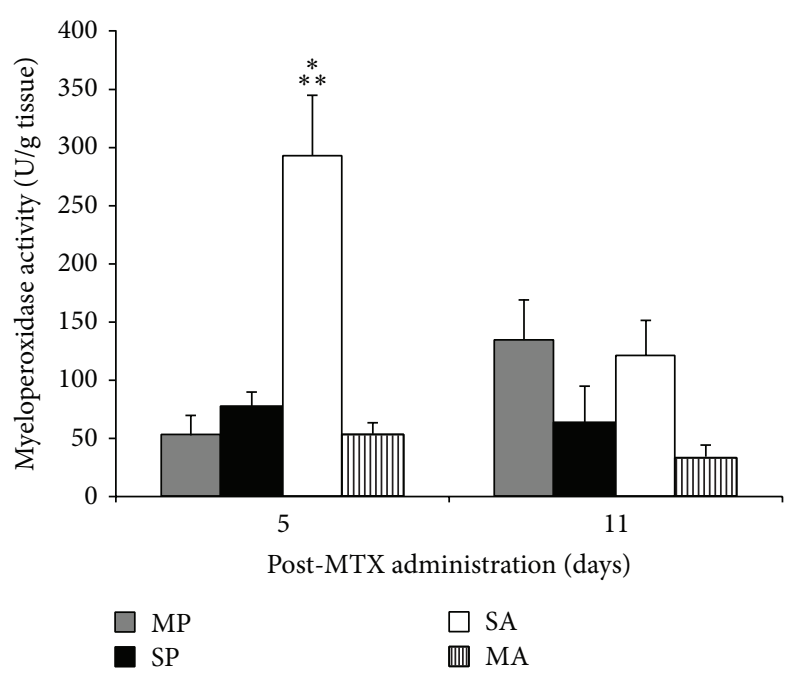

(a)

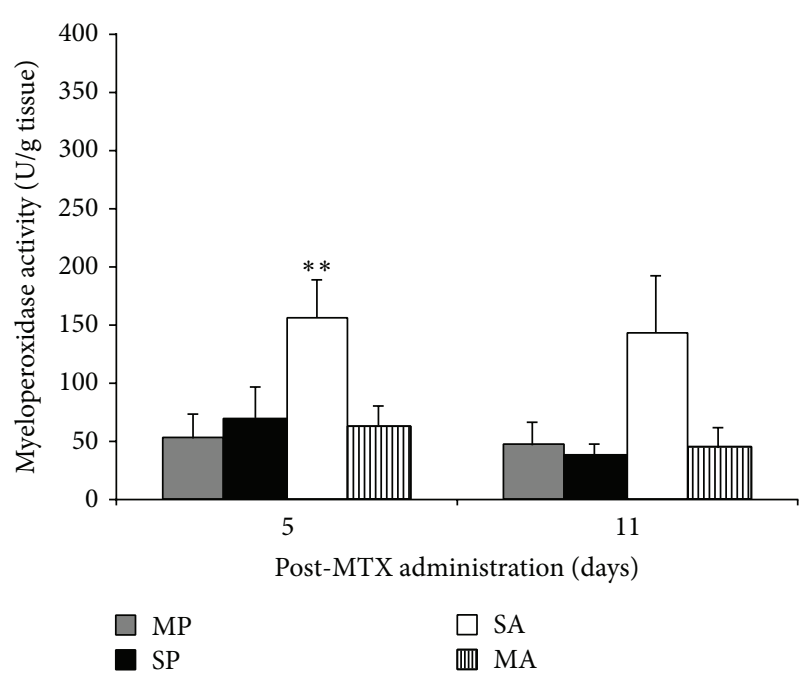

(b)

Figure 2: MPO activity (U/g tissue) in the duodenum (a) and jejunum (b) of C57BL/6 mice treated with PS, PM, AS, and MA ( $n=8 /$ group). $* *$ indicates significant differences $(P<0.01)$ compared to all other groups on $\mathrm{d} 5$. $*$ denotes significance $(P<0.05)$ compared to $P M$ on $\mathrm{d} 11$.

USA) followed by $4 \%$ haemoglobin to mop up excess ${ }^{109} \mathrm{Cd}$. The supernatant was then removed, transferred into a liquid scintillation vial, capped, and analysed using a gamma counter (1282 CompuGamma LRB Wallac, Finland). Results were expressed as nmol of $\mathrm{Cd}$ bound/g of wet weight.

2.8. Histological Assessment. Intestinal samples were fixed in $10 \%$ formalin, processed, embedded in paraffin wax, and then cut and stained with hematoxylin and eosin (H\&E) as described previously [25]. Histological assessments were analysed using light microscopes (Olympus, Tokyo, Japan) with an attached video camera (Sony Corporation Tokyo Japan). For villus height and crypt depth, 40 measurements were taken using Image Pro Plus 5.1 software (Media Cybernetics, Maryland, USA). Histological scores were analysed using 11 histological parameters consisting of villus height, crypt depth ratio, enterocyte disruption, goblet cell numbers, mitotic figures, crypt disruption, crypt cell disruption, crypt abscess formation, lymphocytic and polymorphonucleocyte infiltration, capillary and lymphatic dilatation, submucosal thickness, and muscularis thickening. Each score was given a value of $0-3$ and combined for an overall score.

2.9. Data Analysis. The results were analyzed using SigmaPlot 12.0 (Systat Software, Inc, USA). Two-way ANOVAs with Tukey post hoc tests were used to analyze the results for significance. Statistical significance was considered if $P<0.05$. Data was expressed as mean \pm SEM, unless otherwise stated.

\section{Results}

3.1. Disease Activity Index (DAI). The DAI of the AM group showed no significant difference compared to all other groups throughout the experimental protocol (Table 1). However, the PM group has a significantly $(P=0.01)$ higher DAI compared to the PS group on d 3 and d 5 (Table 1).

3.2. $M P O$ Activity. The AM group showed reduced MPO levels by $59 \%$ compared to mice in the PM group $(P<0.05)$ on $\mathrm{d} 5$ (maximal damage) (Figure 2). The mice in the PM group showed increased MPO levels compared to the AS and PS groups within $\mathrm{d} 5$ in jejunum by $60 \%$ and the duodenum by $33 \%$ (Figure 2 ).

3.3. MT Levels. The AM group showed a significant $(P<$ 0.05 ) reduction in MT levels by $64 \%$ compared to the mice in the PM group on $\mathrm{d} 5$ (maximal damage) (Figure 3). The PM group showed an increase in MT levels compared to the AS and PS groups within d 5 in all three intestinal segments (Figure 3). The mice in the AM group showed a reduction $(P<0.05)$ in MT levels by $22 \%$ compared to the PM group in the duodenum and jejunum only, on d 11 (Figure 3). 


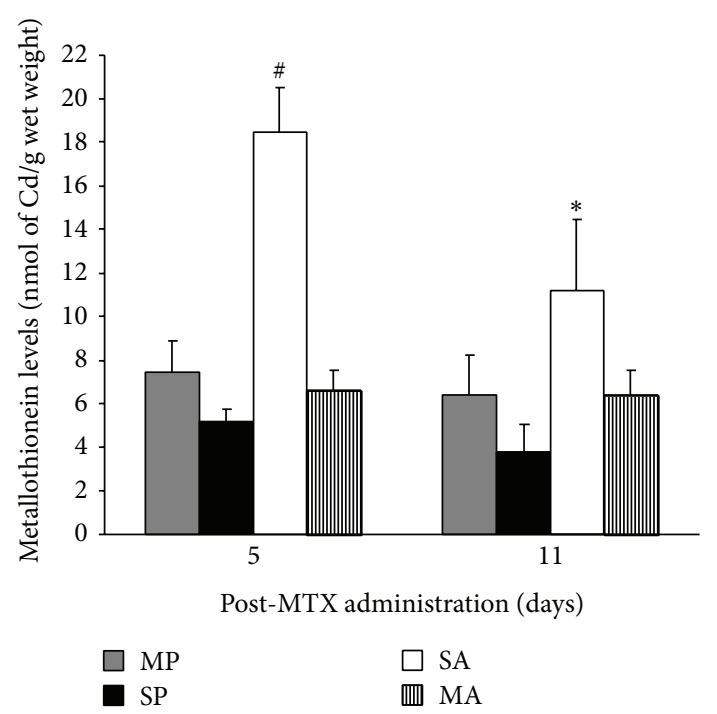

(a)

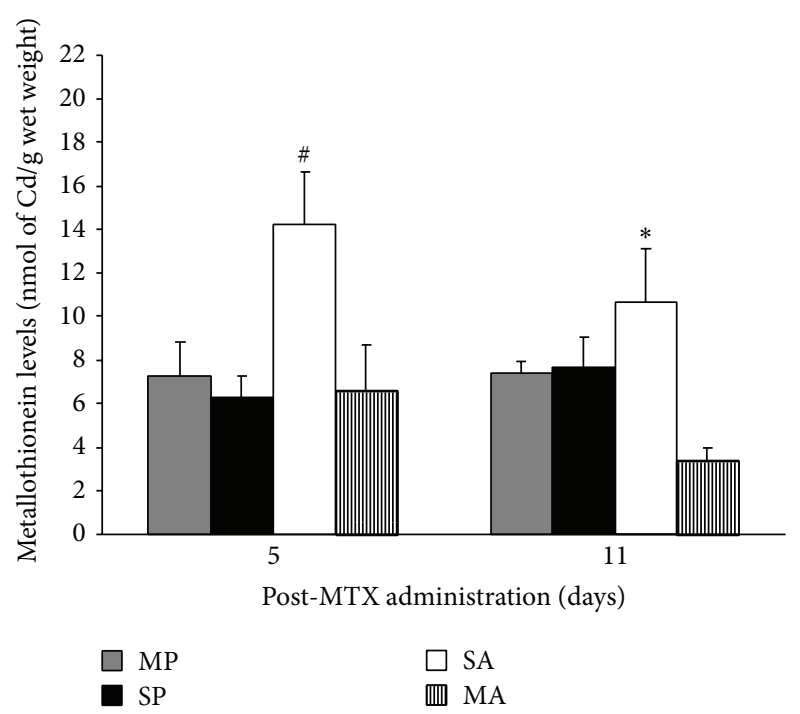

(b)

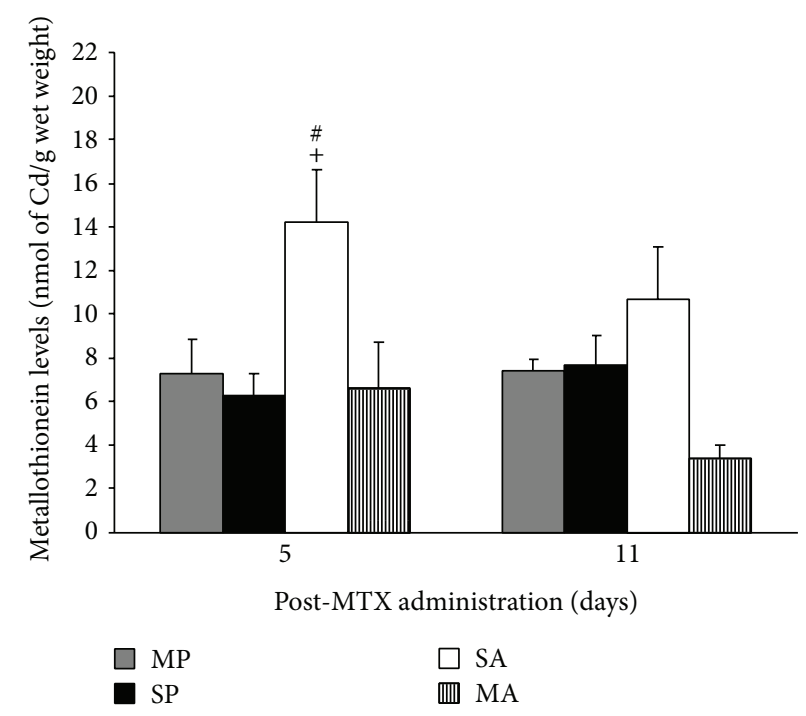

(c)

Figure 3: MT levels (nmol Cd bound/g wet weight) in the duodenum (a), jejunum (b), and ileum (c) of C57BL/6 mice treated with PS, $\mathrm{PM}, \mathrm{AS}$, and MA $(n=8$ /group). \# indicates significant differences $(P<0.01)$ compared to all other groups on $\mathrm{d} 5 . *$ denotes significance $(P<0.05)$ compared to AS on d 11. + indicates significance $(P=0.03)$ compared to d 5 and $\mathrm{d} 11$ in PM group.

3.4. Villus Height and Crypt Depth. The PM group showed a significant increase in crypt depth and decrease in villus height on $\mathrm{d} 5$ compared to all other groups in the duodenum and jejunum (Figures 4 and 5). Crypt depth and villus height were significantly $(P<0.05)$ increased by 49,51 , and $33 \%$ and decreased by 19,38 , and $10 \%$, respectively, in the PM group on $\mathrm{d} 5$ compared to $\mathrm{d} 11$ (Figures 4 and 5).

3.5. Histological Severity Scores. PM treated mice had a significantly increased ( $88 \%$ ) histological severity score on d 5 compared to all other groups (Table 2; Figure 6). Histological severity scores were significantly $(P<0.05)$ decreased in the PM treated group on $\mathrm{d} 11$ compared to $\mathrm{d} 5$ (Table 2; Figure 6). Mice treated with PM showed a decreased infiltration of inflammatory cells and increased mitotic cells present as well as a reduction in damage to cell structure compared to AM and $\mathrm{PB}$ treated mice on $\mathrm{d} 5$ (Figure 6).

\section{Discussion}

The current study investigated the protective effect of acteoside, a herbal substance, on a mouse model of intestinal mucositis. Acteoside is known for its antioxidative properties as well as its ability to reduce inflammation in multiple tissues [19]. The administration of 3 consecutive doses of MTX (daily) induced a mild mucositis which was observed on $\mathrm{d} 5$ as indicated by increased MPO activity and histological severity scores and this is consistent with previous studies $[3-5,25]$.

DAI is indicative of the severity of intestinal damage and mice treated with acteoside showed a trend towards a 
TABLE 2: Histological severity scores for C57BL/6 mice ( $n=8 /$ group) treated with PS, PM, AS, and MA.

\begin{tabular}{|c|c|c|c|c|c|c|}
\hline \multirow{2}{*}{ Treatment } & \multicolumn{2}{|c|}{ Duodenum } & \multicolumn{2}{|c|}{ Jejunum } & \multicolumn{2}{|c|}{ Ileum } \\
\hline & d 5 & $\mathrm{~d} 11$ & d 5 & $\mathrm{~d} 11$ & d 5 & $\mathrm{~d} 11$ \\
\hline PS & $2.5(2,5)$ & $3(1,6)$ & $2.5(1,4)$ & $5(2,9)$ & $3(1,5)$ & $1(1,5)$ \\
\hline AS & $2.5(1,3)$ & $2(1,6)$ & $3(2,7)$ & $3(2,4)$ & $1.5(1,4)$ & $2(0,2)$ \\
\hline PM & $13(2,16)^{*}$ & $2(1,4)^{\#}$ & $13(10,18)^{*}$ & $6(2,10)^{\#}$ & $13(7,15)^{*}$ & $1(0,3)^{\#}$ \\
\hline MA & $3(2,5)$ & $2.5(1,6)$ & $2.5(2,4)$ & $3(1,8)$ & $1(1,3)$ & $1(1,4)$ \\
\hline
\end{tabular}

Data was expressed as median and range in parenthesis. ${ }^{*}$ Statistical significance compared to all other groups $(P<0.01)$ on $\mathrm{d} 5 .{ }^{\#}$ Statistical significance $(P<$ 0.01) compared to d 5 . PS = PBS + saline, AS = acteoside + saline, $\mathrm{PM}=\mathrm{PBS}+\mathrm{MTX}$, and MA = MTX + acteoside.

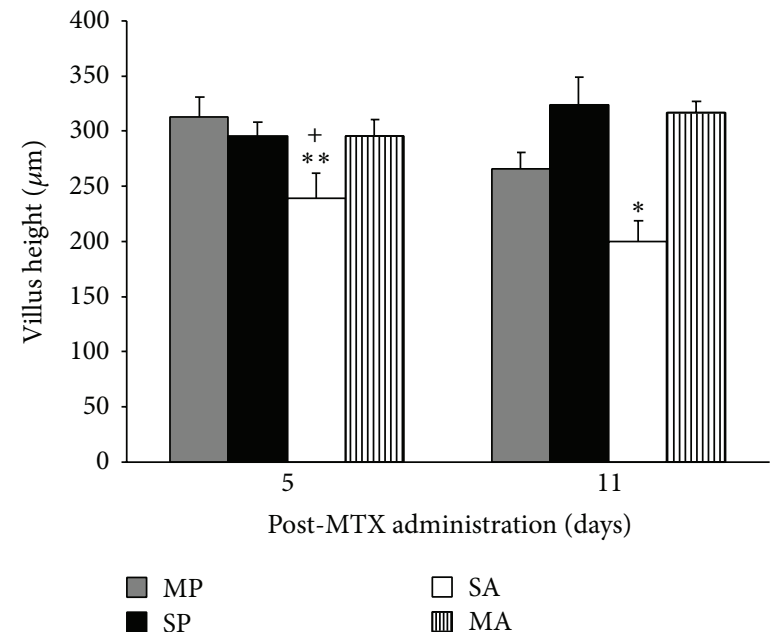

(a)
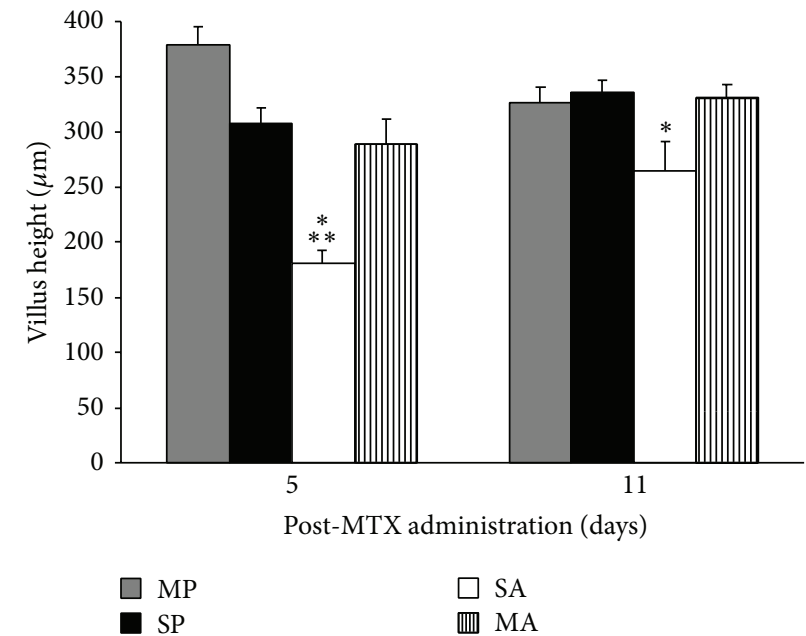

(b)

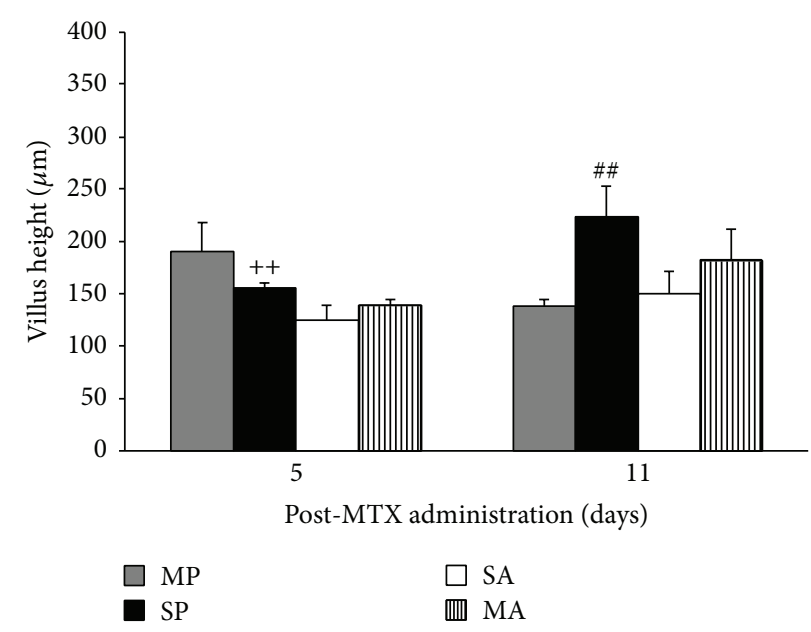

(c)

Figure 4: Villus height $(\mu \mathrm{m})$ in the duodenum (a), jejunum (b), and ileum (c) of C57BL/6 mice treated with PS, PM, AS, and MA. * denotes significant difference $(P<0.05)$ compared to all other groups. $* *$ denotes significant difference $(P=0.027)$ compared to day $11 .+$ indicates significance $(P<0.01)$ compared to PS on $\mathrm{d} 5$. \#\# indicates significant difference compared to PS and PM groups on $\mathrm{d} 11 .++$ indicates significant difference $(P=0.015)$ compared to $\mathrm{d} 11$.

reduction in the severity of clinical symptoms caused by MTX. The trend represented in the DAI scores was consistent with the results observed from the severity scores suggesting that the DAI may be a good indicator of mucosal damage. Furthermore, we have shown that acteoside may be protective against MTX-induced mucositis as supported by our data of reduced severity scores, crypt depth, MPO activity, and MT levels.

Based on our histological severity score parameters, it was observed that degradation of the cellular structure and shape of the cells present in the mucosal lining was reduced. No noticeable changes were observed in mucosal thickening or 


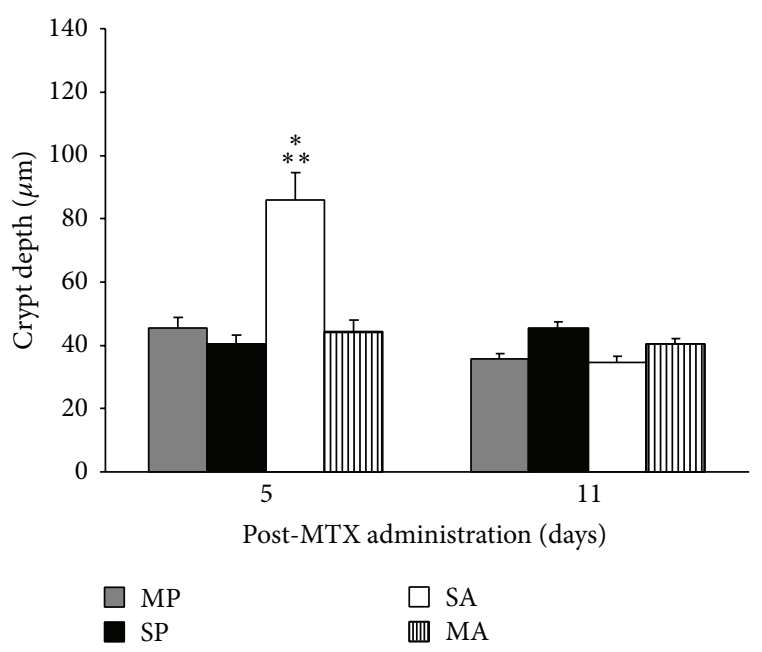

(a)

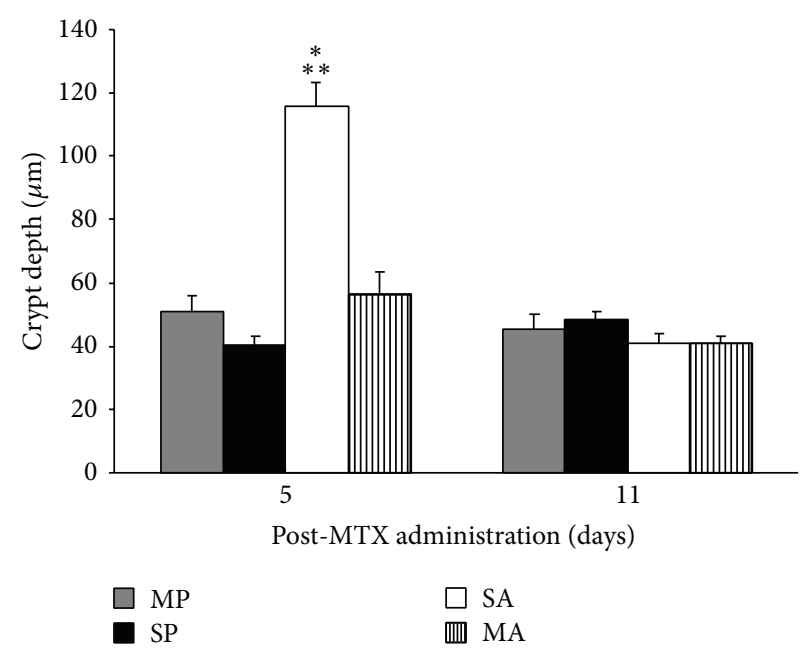

(b)

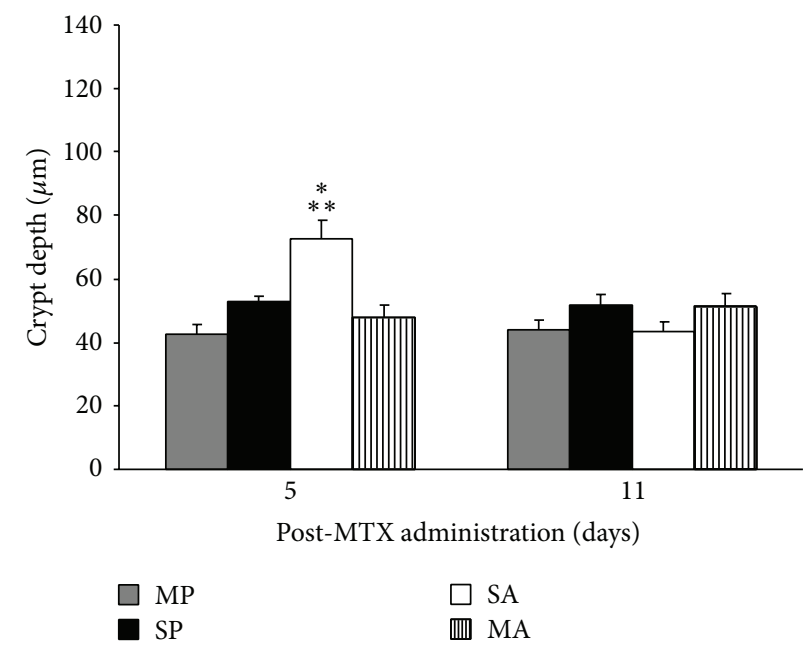

(c)

Figure 5: Crypt depth $(\mu \mathrm{m})$ in the duodenum (a), jejunum (b), and ileum (c) of C57BL/6 mice treated with PS, PM, AS, and MA. * denotes significant difference $(P<0.01)$ compared to all other groups within d $5 . * *$ denotes significant difference $(P<0.01)$ compared to d 11 .

vascularisation. The dividing cells in the base of the crypts were protected and the number of inflammatory cells present in the mucosa were reduced, consistent with previous studies $[21,25]$. The severity scores suggested that acteoside may have a protective action on the small intestine mucosal lining. Furthermore, crypt depth on d 5 was reduced while villus height was increased in mice given acteoside. This indicated the possibility of a protective action on the cells in the villus and crypts. This may be due to a decrease in the production of TNF- $\alpha$ which, in turn, reduces the inflammation by preventing apoptosis of the stem cells in the crypts [26]. We have also shown that the administration of acteoside reduced MPO activity in mice treated with MTX indicating that acteoside may reduce MTX-induced inflammation.

The administration of acteoside markedly reduced MT levels in mice treated with MTX compared to mice treated with MTX alone. MT, an intracellular heavy metal binding, cystine rich ligand, has the ability to bind ROS [27]. The findings suggest that the endogenous MT may have been used to bind and break down ROS associated with MTX-induced mucositis which has been postulated to be the primary reason for reducing inflammation [28]. However, it is unclear how this is achieved; interestingly, we found that acteoside alone did not induce MT levels and there are no supporting studies indicating that acteoside upregulates MT. Further research into this pathway is warranted.

However, it has been shown that acteoside reduces levels of TNF- $\alpha$ [26], cell mediated apoptosis, and the production of ROS is regulated by TNF- $\alpha$ [29-34]. The reduction of TNF- $\alpha$ by acteoside means that local inflammation and cell mediated apoptosis are reduced by the presence of acteoside. This forms a possible mechanism for the function of acteoside in reducing intestinal damage by MTX. Acteoside is known to have antiapoptotic properties [18]. Its ability to inhibit the production of ROS inside cells enables it to prevent the apoptosis of cells [14]. This process suggests that acteoside is 


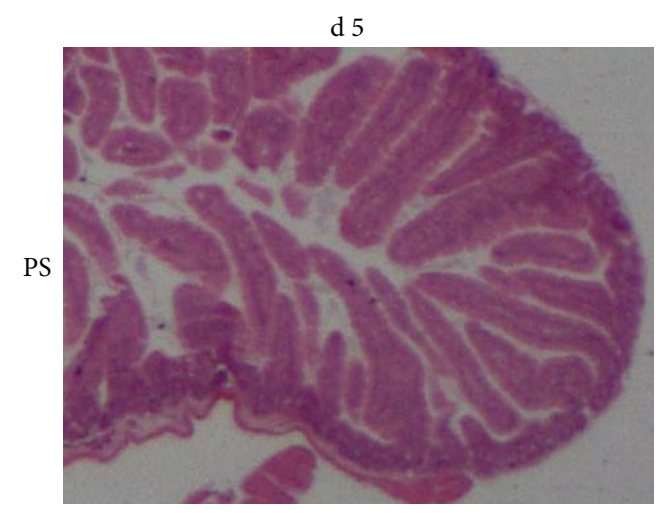

(a)

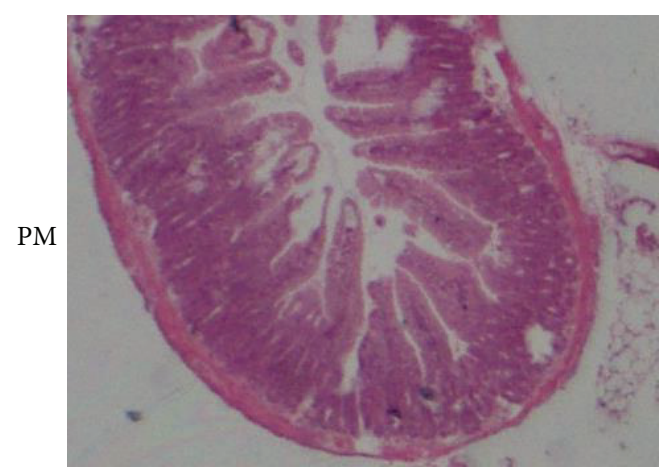

(c)

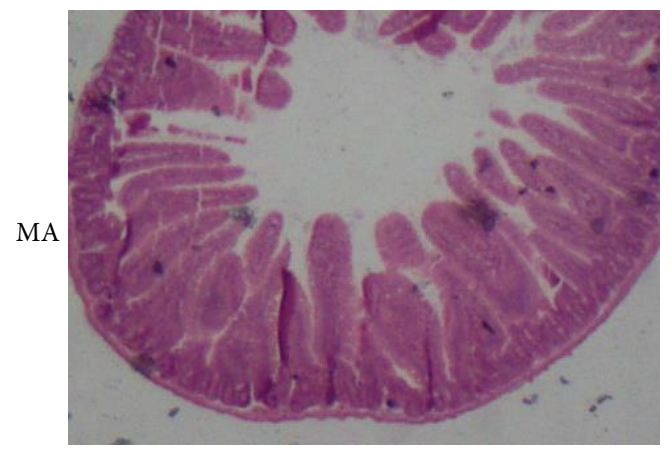

(e)

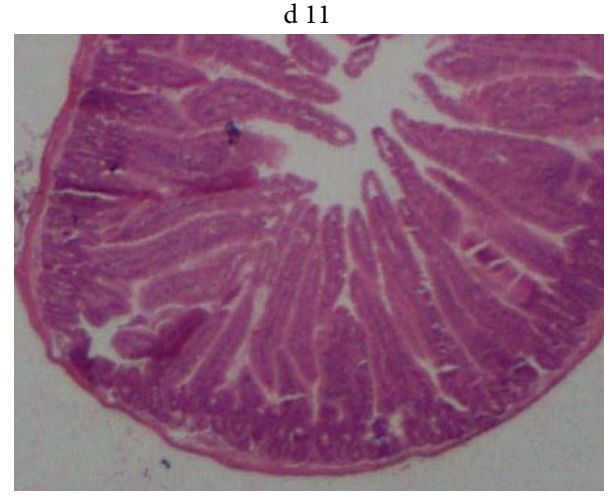

(b)

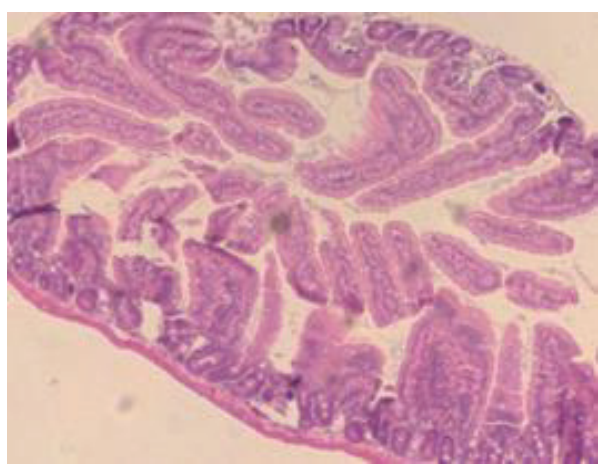

(d)

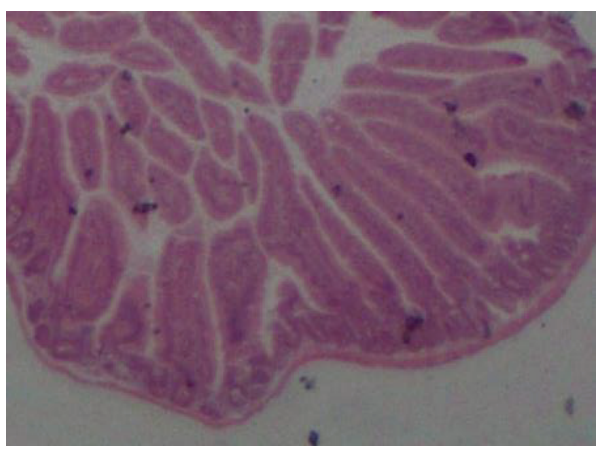

(f)

FIGURE 6: Histological sections of the jejunum stained with H\&E taken at 20x magnification. Images (a) and (b) represent the PS group (control) for d 5 and d 11, respectively, with normal villi and crypts. Images (c) and (d) represent the PM group (MTX-treated) for d 5 and d 11, respectively; the administration of MTX was characterized by atrophy of the villi, increased crypt depth, and enterocyte disruption, predominantly on d 5. Images (e) and (f) represent the MA group (MTX-treated + acteoside) for d 5 and d 11, respectively; the administration of acteoside administration prevented villus and crypt damage on $\mathrm{d} 5$ and $\mathrm{d} 11$.

able to reduce TNF- $\alpha$ levels within the cell, which is a marker essential for cell mediated death $[14,26]$. This implies that the preventative treatment of mice with acteoside may inhibit the initial lysing of basal stem cells in the small intestine in the short term preventing the initial change in tissue response.

The findings from the present study did not show an improvement in recovery rate with respect to MTX-induced damage with the administration of acteoside. This may be expected as acteoside has been shown to partially prevent MTX-induced damage on d 5, and an increased recovery rates may not be observable. It is possible that the protection to the mucosal lining by acteoside was sufficient in the early phase of damage so that recovery rate from damage played a minor role. However, it is possible that acteoside functions in such a way as to prevent increased inflammation but has no direct effect on enhancing the rate of recovery of cells that have already been damaged.

In conclusion, mice treated with MTX to induce mucositis showed reduced levels of damage and inflammation to the mucosal lining when given acteoside prophylactically. Acteoside may be protective against MTX-induced small intestinal damage, by a couple of mechanisms, firstly increasing 
mitotic cells, thereby increasing cell proliferation, and secondly reducing inflammatory cell numbers as indicated by decreased MPO activity. Acteoside appears to be a promising alternative therapeutic approach for treating chemotherapyinduced mucositis.

\section{Conflict of Interests}

The authors have no conflict of interests to declare.

\section{Authors' Contribution}

Cuong D. Tran and Stamatiki Kritas designed the experiment and Daniel Reinke conducted the experiments and did the analyses. All authors contributed to the design and review of the paper.

\section{Acknowledgments}

The authors would like to acknowledge Ms. Kerry Lymn for providing technical assistance in the animal trials and histology. This study was supported by the Gastroenterology Departmental Fund at the Women's and Children's Health Network.

\section{References}

[1] J.-L. Pico, A. Avila-Garavito, and P. Naccache, "Mucositis: its occurrence, consequences, and treatment in the oncology setting," Oncologist, vol. 3, no. 6, pp. 446-451, 1998.

[2] S. T. Sonis and K. A. Costello, "A database for mucositis induced by cancer chemotherapy," European Journal of Cancer-Part B: Oral Oncology, vol. 31, no. 4, pp. 258-260, 1995.

[3] B. A. E. de Koning, J. M. van Dieren, D. J. Lindenbergh-Kortleve et al., "Contributions of mucosal immune cells to methotrexateinduced mucositis," International Immunology, vol. 18, no. 6, pp. 941-949, 2006.

[4] S. T. Sonis, "Mucositis: the impact, biology and therapeutic opportunities of oral mucositis," Oral Oncology, vol. 45, no. 12, pp. 1015-1020, 2009.

[5] R. M. Logan, A. M. Stringer, J. M. Bowen et al., "The role of pro-inflammatory cytokines in cancer treatment-induced alimentary tract mucositis: pathobiology, animal models and cytotoxic drugs," Cancer Treatment Reviews, vol. 33, no. 5, pp. 448-460, 2007.

[6] N. Boukhettala, J. Leblond, S. Claeyssens et al., "Methotrexate induces intestinal mucositis and alters gut protein metabolism independently of reduced food intake," The American Journal of Physiology: Endocrinology and Metabolism, vol. 296, no. 1, pp. E182-E190, 2009.

[7] M. R. Posner and R. I. Haddad, "Novel agents for the treatment of mucositis," Journal of Supportive Oncology, vol. 5, no. 9, pp. 33-39, 2007.

[8] E. B. Rubenstein, D. E. Peterson, M. Schubert et al., "Clinical practice guidelines for the prevention and treatment of cancer therapy-induced oral and gastrointestinal mucositis," Cancer, vol. 100, no. S9, pp. 2026-2046, 2004.

[9] D. M. Keefe, S. T. Sonis, and J. M. Bowen, "Emerging drugs for chemotherapy-induced mucositis," Expert Opinion on Emerging Drugs, vol. 13, no. 3, pp. 511-522, 2008.
[10] D. M. Keefe, M. M. Schubert, L. S. Elting et al., "Updated clinical practice guidelines for the prevention and treatment of mucositis," Cancer, vol. 109, no. 5, pp. 820-831, 2007.

[11] L. Negri, R. Lattanzi, E. Giannini et al., "Impaired nociception and inflammatory pain sensation in mice lacking the prokineticin receptor PKR1: focus on interaction between PKR1 and the capsaicin receptor TRPV1 in pain behavior," The Journal of Neuroscience, vol. 26, no. 25, pp. 6716-6727, 2006.

[12] T. H. Wright, R. Yazbeck, K. A. Lymn et al., "The herbal extract, Iberogast, improves jejunal integrity in rats with 5-Fluorouracil (5-FU)-induced mucositis," Cancer Biology and Therapy, vol. 8, no. 10, pp. 923-929, 2009.

[13] W. T. Y. Loo, L. Jin, L. W. C. Chow, M. N. B. Cheung, and M. Wang, "Rhodiola algida improves chemotherapy-induced oral mucositis in breast cancer patients," Expert Opinion on Investigational Drugs, vol. 19, no. 1, pp. S91-S100, 2010.

[14] A. M. Díaz, M. J. Abad, L. Fernández, A. M. Silván, J. de Santos, and P. Bermejo, "Phenylpropanoid glycosides from Scrophularia scorodonia: in vitro anti-inflammatory activity," Life Sciences, vol. 74, no. 20, pp. 2515-2526, 2004.

[15] B. Konuklugil and Ö. Bahadir, "Phenylpropanoid glycosides from Linum olympicum," Turkish Journal of Chemistry, vol. 28, no. 6, pp. 741-744, 2004.

[16] Y. P. Hwang, H. G. Kim, J. H. Choi et al., "Acteoside inhibits PMA-induced matrix metalloproteinase-9 expression via CaMK/ERK- and JNK/NF- $\kappa \mathrm{B}$-dependent signaling," Molecular Nutrition \& Food Research, vol. 55, supplement 1, pp. S103S116, 2011.

[17] A. Güvenç, Y. Okada, E. K. Akkol, H. Duman, T. Okuyama, and I. Çaliş, "Investigations of anti-inflammatory, antinociceptive, antioxidant and aldose reductase inhibitory activities of phenolic compounds from Sideritis brevibracteata," Food Chemistry, vol. 118, no. 3, pp. 686-692, 2010.

[18] M. Hausmann, F. Obermeier, D. H. Paper et al., "In vivo treatment with the herbal phenylethanoid acteoside ameliorates intestinal inflammation in dextran sulphate sodium-induced colitis," Clinical and Experimental Immunology, vol. 148, no. 2, pp. 373-381, 2007.

[19] Q. Xiong, K. Hase, Y. Tezuka, T. Namba, and S. Kadota, "Acteoside inhibits apoptosis in D-Galactosamine and lipopolysaccharide- induced liver injury," Life Sciences, vol. 65, no. 4, pp. 421-430, 1999.

[20] T. Genovese, I. Paterniti, E. Mazzon et al., "Efficacy of treatment with verbascoside, biotechnologically produced by Syringa vulgaris plant cell cultures in an experimental mice model of spinal cord trauma," Naunyn-Schmiedeberg's Archives of Pharmacology, vol. 382, no. 4, pp. 331-345, 2010.

[21] J. Y. Lee, E.-R. Woo, and K. W. Kang, "Inhibition of lipopolysaccharide-inducible nitric oxide synthase expression by acteoside through blocking of AP-1 activation," Journal of Ethnopharmacology, vol. 97, no. 3, pp. 561-566, 2005.

[22] C. D. Tran, S. Sundar, and G. S. Howarth, "Dietary zinc supplementation and methotrexate-induced small intestinal mucositis in metallothionein-knockout and wild-type mice," Cancer Biology and Therapy, vol. 8, no. 17, pp. 1662-1667, 2009.

[23] A. Dahlqvist, "Method for assay of intestinal disaccharidases," Analytical Biochemistry, vol. 7, no. 1, pp. 18-25, 1964.

[24] D. L. Eaton and B. F. Toal, "Evaluation of the Cd/hemoglobin affinity assay for the rapid determination of metallothionein in biologival tissues," Toxicology and Applied Pharmacology, vol. 66, no. 1, pp. 134-142, 1982. 
[25] G. S. Howarth, G. L. Francis, J. C. Cool, X. Xu, R. W. Byard, and L. C. Read, "Milk growth factors enriched from cheese whey ameliorate intestinal damage by methotrexate when administered orally to rats," The Journal of Nutrition, vol. 126, no. 10, pp. 2519-2530, 1996.

[26] R. Medeiros, R. D. S. Prediger, G. F. Passos et al., "Connecting TNF- $\alpha$ signaling pathways to iNOS expression in a mouse model of Alzheimer's disease: relevance for the behavioral and synaptic deficits induced by amyloid $\beta$ protein," Journal of Neuroscience, vol. 27, no. 20, pp. 5394-5404, 2007.

[27] D. M. Templeton and M. G. Cherian, "Toxicological significance of metallothionein," Methods in Enzymology, vol. 205, pp. 11-24, 1991.

[28] J. Abel and N. de Ruiter, "Inhibition of hydroxyl-radicalgenerated DNA degradation by metallothionein," Toxicology Letters, vol. 47, no. 2, pp. 191-196, 1989.

[29] M. Fotin-Mleczek, F. Henkler, A. Hausser et al., “Tumor necrosis factor receptor-associated factor (TRAF) 1 regulates CD40induced TRAF2-mediated NF- $\kappa \mathrm{B}$ activation," The Journal of Biological Chemistry, vol. 279, no. 1, pp. 677-685, 2004.

[30] K. Hayashi, T. Nagamatsu, M. Ito, H. Yagita, and Y. Suzuki, "Acteoside, a component of Stachys sieboldii MIQ, may be a promising antinephritic agent (3): effect of acteoside on expression of intercellular adhesion molecule-1 in experimental nephritic glomeruli in rats and cultured endothelial cells," The Japanese Journal of Pharmacology, vol. 70, no. 2, pp. 157-168, 1996.

[31] Q. Xiong, Y. Tezuka, T. Kaneko et al., "Inhibition of nitric oxide by phenylethanoids in activated macrophages," European Journal of Pharmacology, vol. 400, no. 1, pp. 137-144, 2000.

[32] L. Speranza, S. Franceschelli, M. Pesce et al., "Antiinflammatory effects in THP-1 cells treated with verbascoside," Phytotherapy Research, vol. 24, no. 9, pp. 1398-1404, 2010.

[33] Y. Al-Gindan, M. Shawarby, A. Noto, and C. G. Taylor, "Intestinal inflammation in rats induces metallothionein in colonic submucosa," Journal of Clinical Biochemistry and Nutrition, vol. 44, no. 2, pp. 131-141, 2009.

[34] M. Brüwer, K. W. Schmid, K. A. Metz, C. F. Krieglstein, N. Senninger, and G. Schürmann, "Increased expression of metallothionein in inflammatory bowel disease," Inflammation Research, vol. 50, no. 6, pp. 289-293, 2001. 


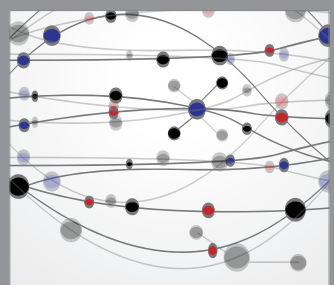

The Scientific World Journal
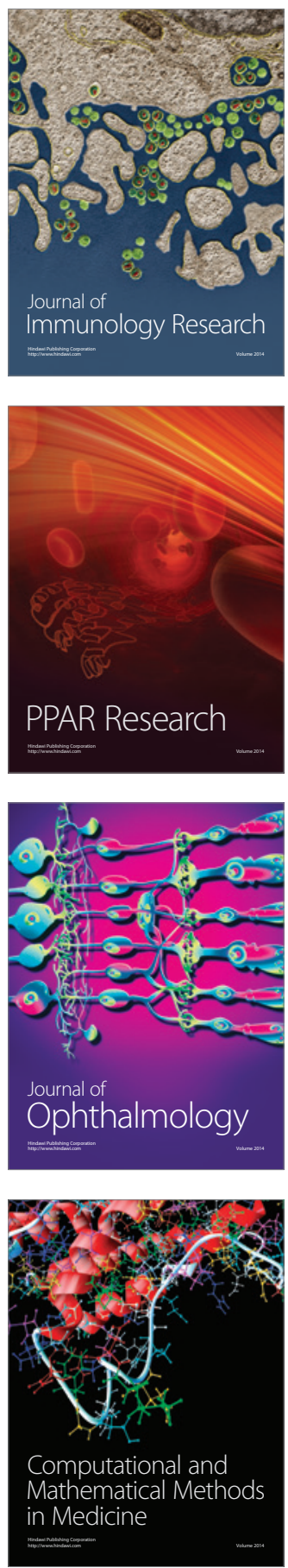

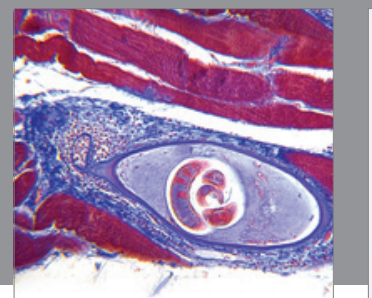

Gastroenterology

Research and Practice
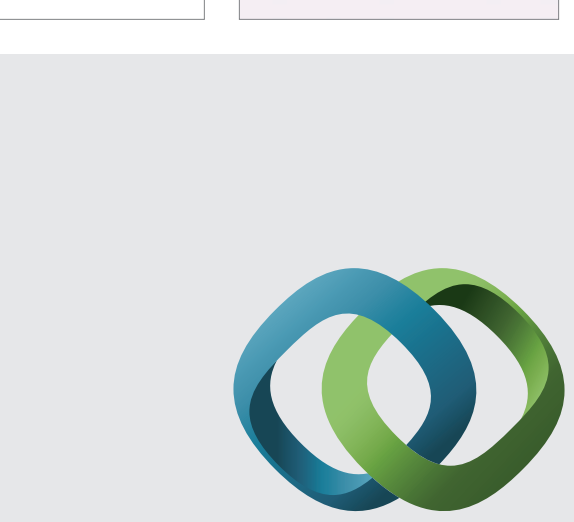

\section{Hindawi}

Submit your manuscripts at

http://www.hindawi.com
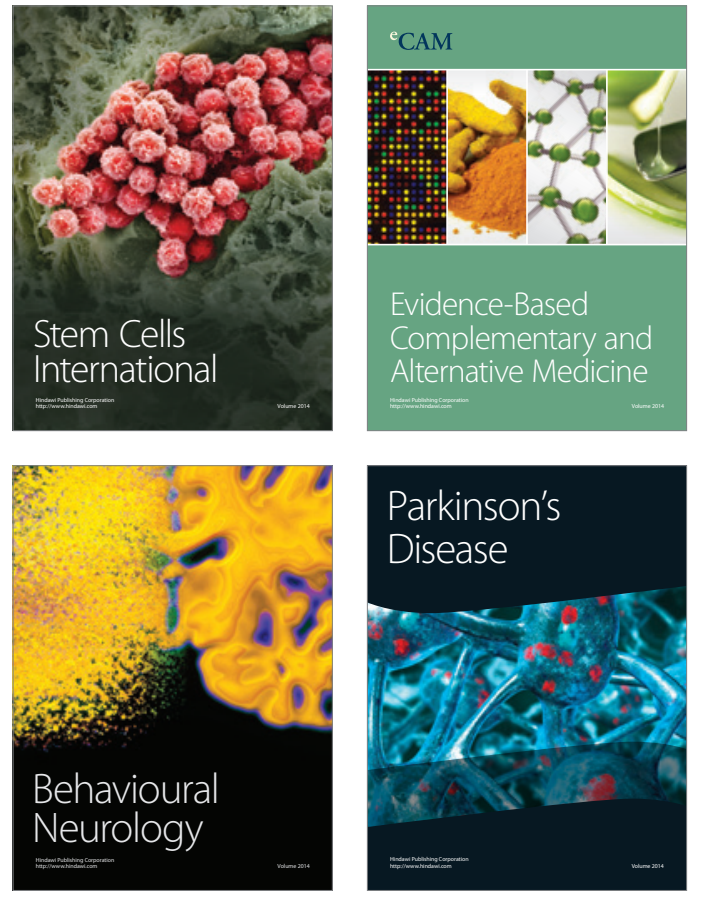
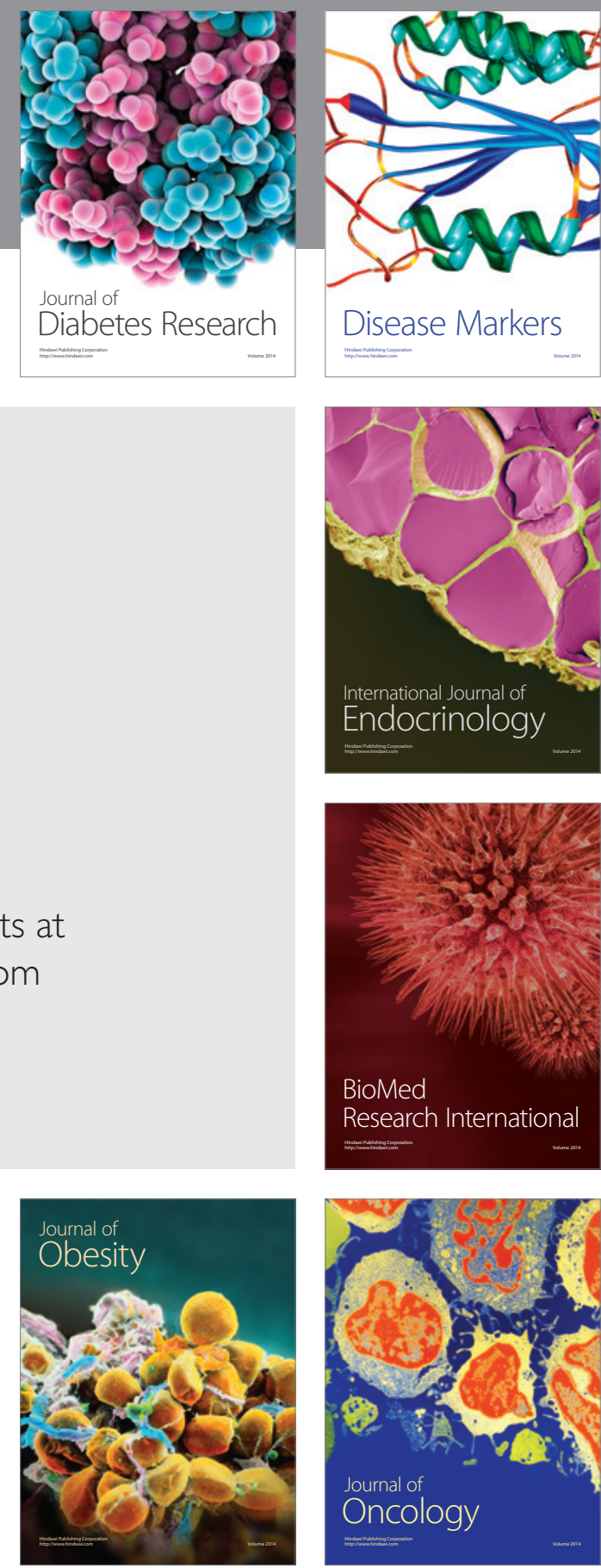

Disease Markers
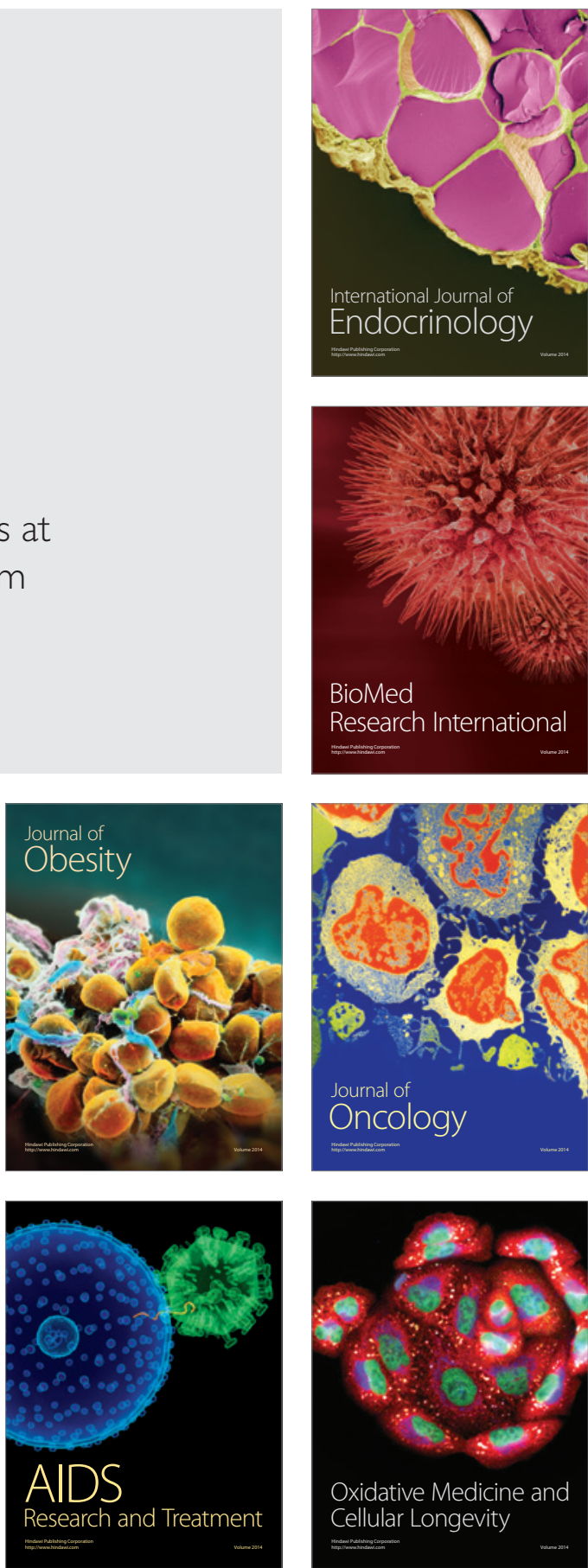